\title{
Cuidados paliativos: a comunicação como ferramenta no tratamento de pacientes
}

\section{idosos oncológicos}

\author{
Palliative care: communication as a tool in the treatment of elderly oncology patients \\ Cuidados paliativos: la comunicación como herramienta en el tratamiento del paciente anciano \\ oncológico
}

Recebido: 17/08/2021 | Revisado: 26/08/2021 | Aceito: 31/08/2021 | Publicado: 03/09/2021

Luciana da Silva dos Santos

ORCID: https://orcid.org/0000-0002-5828-8372 Instituto Brasileiro de Medicina de Reabilitação, Brasil E-mail: lucianaguinado2016@gmail.com

Claudia Beatriz Assumpção de Oliveira

ORCID: https://orcid.org/0000-0003-2713-2006 Instituto Brasileiro de Medicina de Reabilitação, Brasil E-mail: cbdeoliveira@gmail.com

Allan Carlos Mazzoni Lemos

ORCID: https://orcid.org/0000-0002-7818-9478 Instituto Brasileiro de Medicina de Reabilitação, Brasil E-mail: allanmazzoni@gmail.com

\begin{abstract}
Resumo
Objetivo: O presente estudo objetivou analisar a importância das estratégias de comunicação no atendimento humanizado em Cuidados Paliativos aos pacientes oncológicos, então, estabeleceu-se como questão norteadora: Quais estratégias de comunicação na relação entre profissionais de enfermagem, pacientes oncológicos e seus familiares nos cuidados paliativos? Metodologia: Trata-se de uma pesquisa de revisão sistemática da literatura, realizada entre os meses de janeiro a maio de 2020, na Biblioteca Virtual da Saúde, filtrando as seguintes bases de dados: Scientific Electronic Library Online, Literatura Latino-Americana e do Caribe em Ciências da Saúde, Base de dados em Enfermagem e Medical Literature Analysis and Retrieval System Online, por intermeio dos Descritores em Ciências da Saúde: Cuidados Paliativos; Enfermagem; Idoso; Câncer e Comunicação. Como critérios de inclusão, definiu-se estudos publicados entre os anos de 2015 a 2020, com textos completos. Já os de exclusão foram estudos duplicados e estudos com a população infantil. Resultados: Obteve-se a totalidade de 13 artigos científicos publicados nos últimos 5 anos e 1 livro. Estes foram_analisados e expostos em uma tabela com 3 tópicos: título, autor/ano de publicação e conclusão. Conclusão: Observou-se que as estratégias de comunicação devem ser desenvolvidas baseadas no cuidado humanizado e que a capacitação do profissional é um fator imprescindível para que assistência de enfermagem seja bem-sucedida.
\end{abstract}

Palavras-chave: Cuidados paliativos; Enfermagem; Idoso; Câncer; Comunicação.

\begin{abstract}
Objective: This study aimed to analyze the importance of communication strategies in humanized care in palliative care for cancer patients, so the guiding question was established: What communication strategies in the relationship between nursing professionals, cancer patients and their families in care palliatives? Methodology: This is a systematic literature review research, carried out between January and May 2020, in the Virtual Health Library, filtering the following databases: Scientific Electronic Library Online, Latin American and Caribbean Literature in Health Sciences, Database in Nursing and Medical Literature Analysis and Retrieval System Online, through the Descriptors in Health Sciences: Palliative Care; Nursing; Old man; Cancer and Communication. As inclusion criteria, studies published between the years 2015 to 2020 were defined, with full texts. The exclusion ones were duplicated studies and studies with the child population. Results: A total of 13 scientific articles published in the last 5 years and 1 book were obtained. These were analyzed and displayed in a table with 3 topics: title, author/year of publication and conclusion. Conclusion: It was observed that communication strategies must be developed based on humanized care and that professional training is an essential factor for successful nursing care.
\end{abstract}

Keywords: Palliative care; Nursing; Old man; Cancer; Communication.

\section{Resumen}

Objetivo: Este estudio tuvo como objetivo analizar la importancia de las estrategias de comunicación en los cuidados humanizados en cuidados paliativos para pacientes con cáncer, por lo que se estableció la pregunta orientadora: ¿Qué 
estrategias de comunicación en la relación entre los profesionales de enfermería, los pacientes con cáncer y sus familias en los cuidados paliativos? Metodología: Se trata de una investigación de revisión sistemática de la literatura, realizada entre enero y mayo de 2020, en la Biblioteca Virtual en Salud, filtrando las siguientes bases de datos: Biblioteca Científica Electrónica en Línea, Literatura Latinoamericana y del Caribe en Ciencias de la Salud, Base de Datos en Enfermería y Análisis de la Literatura Médica. y Sistema de Recuperación en Línea, a través de los Descriptores en Ciencias de la Salud: Cuidados Paliativos; Enfermería; Anciano; Cáncer y comunicación. Como criterios de inclusión se definieron los estudios publicados entre los años 2015 a 2020, con textos completos. Los de exclusión fueron estudios duplicados y estudios con población infantil. Resultados: Se obtuvieron un total de 13 artículos científicos publicados en los últimos 5 años y 1 libro. Estos fueron analizados y exhibidos en una tabla con 3 temas: título, autor / año de publicación y conclusión. Conclusión: Se observó que las estrategias de comunicación deben desarrollarse con base en el cuidado humanizado y que la formación profesional es un factor fundamental para el éxito del cuidado de enfermería.

Palabras clave: Cuidados paliativos; Enfermería; Anciano; Cáncer; Comunicación.

\section{Introdução}

Quando falamos sobre os Cuidados Paliativos (CP), estamos nos referindo a enfermagem e todo o processo que ela engloba: saúde, educação, orientação, apoio emocional, social e atuação do enfermeiro como mediador na relação paciente, família e também para os outros profissionais envolvidos no cuidado. Em 2002 a Organização Mundial de Saúde (OMS), redefiniu os CP, como uma abordagem para melhorar a qualidade de vida dos pacientes com doenças ameaçadoras a continuidade da vida, estendendo os cuidados aos seus familiares, por meio de ações de prevenção e alívio de todo o sofrimento físico, psicológico, social e espiritual. O CP deve ser iniciado a partir do diagnóstico de uma doença com prognóstico desfavorável, necessitando assim de uma equipe multidisciplinar, devido a proposição de prestação de serviços variados (Brandão et al, 2017).

No Brasil, o envelhecimento da população é uma realidade e apesar dos avanços tecnológicos na área da saúde, as doenças crônicas degenerativas continuam sem resultados de melhoras. Entretanto, esses avanços contribuíram para a diminuição da taxa de mortalidade mundial. Nos Estados Unidos, por exemplo, passou de 54 anos, em 1920 para 77,3 anos em 2002, já no Brasil segundo os dados estatísticos do IBGE de 2016, a população brasileira, mudou a idade: de 66, em 1981, para 68,6 anos em 2000. O ser humano conquistou um aumento na expectativa de vida, contudo não houve necessariamente uma melhora de qualidade em relação ao envelhecer e/ou adoecer. Em face a necessidade de cuidados específicos, o CP surge como assistência inovadora desviando o foco do tratamento tradicional baseado na cura, pelo olhar holístico do cuidado através da prevenção dos agravos e controle dos sintomas (Gomes \& Othero, 2016).

Nesta perspectiva, o envelhecimento da população é um grande desafio para o sistema de saúde, pois necessita de ações de promoção específica e prevenção, além ainda de políticas públicas adequadas às suas necessidades, para responder a todas as demandas de um cuidado específico e direcionado às peculiaridades provenientes da idade (Miranda, Mendes \& Silva, 2016).

A Constituição Brasileira (1988) estabeleceu um novo sistema de saúde - Sistema Único de Saúde (SUS), que garante para toda população brasileira, atenção integral a saúde abrangendo três níveis de atendimento: o primário que é a porta de entrada com atendimento na Unidade Básica de saúde; o secundário que é o nível de especialização; e, o terciário alta complexidade. No entanto, apesar de ter havido melhorias significativas, a ocorrência de falhas na assistência é um ponto adverso. Pensando em um serviço seguro, o Ministério da Saúde (MS) em 2013, deu início a protocolos visando diminuir dano físico e/ou psicológico ao paciente. Deste modo, um sistema informatizado na plataforma Web foi criado em prol da segurança e qualidade da assistência (Silva et al, 2016).

Desta forma, para evitar possíveis erros durante o atendimento e a assistência aos pacientes, é importante que o profissional de enfermagem esteja familiarizado com a técnica da informatização, visto que ela é um instrumento que facilita e 
agiliza a troca de informação e comunicação entre profissionais, garantindo a segurança na hora do cuidado (Ferreira et al., 2019).

O câncer é uma doença com uma estimativa mundial crescente, que acomete a qualquer pessoa, não somente as que possuem predisposições hereditárias, mas também as que têm um estilo de vida inadequado em relação a uma vida saudável. Caracterizado pelo crescimento desordenado das células, o câncer causa muito sofrimento, angústia e medo. Segundo o Instituto Nacional do Câncer (INCA), o controle da doença requer, a partir do seu diagnóstico, a inclusão da prestação do CP (Costa, Assunção \& Salles, 2017).

Para os pacientes idosos com diagnóstico de câncer o processo de cuidar é fundamental a humanização, que propõe resgatar a forma de cuidado mais atento e humano. Entretanto, é necessário um comprometimento das relações humanas que viabilizem a responsabilidade, a sensibilidade, a solidariedade, a comunicação e a ética como metas no cuidado ao paciente. Outro ponto importante que podemos ressaltar, é a relação interpessoal, que vai além do toque, do olhar, do saber ouvir, e sim, do trabalho de uma equipe multidisciplinar que fuja dos modelos tradicionais e ofereçam novos modelos e paradigmas no atendimento aos cuidados a esses pacientes. No cuidado de forma humanizada três pontos se destacam: a comunicação, assistência humanizada e o trabalho de uma equipe multidisciplinar. A comunicabilidade entra como fator de elo entre as relações interpessoais, podendo ser de forma verbal e não-verbal. Neste contexto, as relações interpessoais são estabelecidas de forma transparente visando a diminuição das dificuldades, promovendo a empatia e o ambiente inclusivo (Costa, Assunção \& Salles, 2017).

A estruturação da enfermagem como ciência teve início, a partir da elaboração das teorias no processo de cuidar. Graças a essas teorias, a enfermagem hoje, é reconhecida como uma ciência e disciplina com conceitos, princípios e fundamentos que conduzem pensamento e ação prática sistemática. A enfermagem fundamenta a sua prática baseada nos seus metaparadigmas, que são: a) Enfermagem é a ciência do cuidado, por meio da assistência com uma metodologia; b) Pessoa é quem necessita e recebe o cuidado; c) Ambiente é o espaço físico da pessoa, podendo ser o domicílio, o trabalho; d) Saúde é o alvo a ser atendido com a assistência, um bem-estar completo (Franzoi et al, 2016; Merino et al, 2018).

Impende salientar que, a teoria das relações interpessoais de Hildegard Elizabeth Peplau, fundamenta seus estudos na relação enfermeiro/paciente, com o objetivo no desenvolvimento pessoal tanto do paciente como do enfermeiro. Na sua visão os metaparadigmas traduzem a enfermagem como uma ferramenta educativa de aprendizado tanto para o paciente como para o enfermeiro. O enfermeiro deve desenvolver uma relação interpessoal pelo uso da comunicação, utilizando a escuta, o esclarecimento, o diálogo e o ensino. Para atender a assistência de enfermagem foi desenvolvido quatro fases que estão centradas na relação enfermeiro/paciente que ocorrem ao longo do processo terapêutico. A comunicação é a ferramenta de destaque nesta relação colaborativa, na qual o papel do enfermeiro e do paciente se modifica gradualmente com a participação ativa de ambos (Franzoi et al, 2016; Siqueira, 2020).

Hildegard Elizabeth Peplau, Americana da Pensilvânia, enfermeira com formação na escola da Pensilvânia e em psicologia interpessoal, pelo Bennington College, onde conheceu e estudou com Hart Stack Sullivan, experiente médico psiquiatra, que colaborou na construção da teoria de Peplau. Com o passar dos anos Peplau destacou-se na área da psicologia e psiquiatria, recebendo o reconhecimento internacional por seu trabalho (Franzoi et al, 2016).

Seguindo a trajetória da área da saúde, notório se faz mencionar que os CP, que surgiram na Inglaterra, através da enfermeira inglesa, Cecily Saunds. Já no Brasil, o conceito tornou-se popular somente em 1990, a partir do projeto piloto do professor, Tulio de Assis Figueiredo que desenvolveu um curso baseado na filosofia paliativista, na Escola Paulista de Medicina da Universidade Federal de São Paulo (UNIFESP/EPM) (Karine, 2018).

Em 1998, o Hospital da Unidade IV, do Instituto Nacional do Câncer (INCA), foi inaugurado pelo Ministro da Saúde do Brasil, um serviço exclusivo para atender pacientes que se encontravam em cuidados paliativos. Mais tarde, em dezembro 
de 2002, foi inaugurada uma enfermaria específica, para esse cuidado no Hospital do Servidor Público Estadual de São Paulo (HSPE/SP). Contudo, foi em 2009, que o Conselho Federal de Medicina (CFM), incluiu os CP, no código de ética medica com princípios fundamentais. Anualmente, comemora-se no mês de outubro o "Dia do Cuidado Paliativo", desde a parceria da Academia Nacional de Cuidados Paliativos (ANCP) com o World Hospice and Palliative Care Day, no ano de 2002 (Karine, 2018).

É essencial no trabalho de cuidador de idoso portador de câncer, o uso de estratégias de comunicação que irão nortear a prática da assistência, promover a participação dos familiares atenuando o sofrimento físico e psicológico de ambos. Assim, a comunicação interpessoal quando é eficaz, abre espaço para a voz do paciente e também para aproximação com a equipe de cuidado, facilitando atuação do enfermeiro e promovendo estratégias de superação das situações adversas (Galvão, Borges \& Pinho, 2017).

Diante do exposto, emerge a seguinte questão norteadora: quais estratégias de comunicação na relação entre profissionais de enfermagem, pacientes oncológicos e seus familiares nos cuidados paliativos?

Sendo assim, objetivou-se analisar a importância das estratégias de comunicação no atendimento humanizado em Cuidados Paliativos aos pacientes oncológicos.

\section{Metodologia}

Trata-se de uma pesquisa de revisão sistemática da literatura. Para sua realização é necessário uma boa investigação sobre a temática previamente escolhida, de forma controlada e sistemática, através da pesquisa em outros estudos (Ludke \& Andre, 2013).

Ocorreu entre o período de janeiro de 2020 a maio, do mesmo ano. As buscas dos artigos ocorreram nas bases de dados da Scientific Electronic Library Online (SCIELO), Literatura Latino-Americana e do Caribe em Ciências da Saúde (LILACS), Base de dados em Enfermagem (BDENF) e Medical Literature Analysis and Retrieval System Online (MEDLINE).

A primeira busca foi realizada na Biblioteca Virtual em Saúde (BVS), a partir da combinação dos seguintes descritores: Cuidados Paliativos, Enfermagem, Idoso, Câncer e Comunicação. Em seguida, delimitou-se os números com as versões em inglês e português para 1.084 artigos.

Destes, usou-se o método de aplicação de critérios de inclusão e exclusão para os artigos, nos quais os de inclusão foram os publicados no período de 2015 a 2020, com textos completos. Já os artigos de exclusão foram os que estavam duplicados, estudos com a população infantil, estudos publicados anteriormente à 2015 e por isto não se adequavam ao propósito da pesquisa, restando assim um total de 75 artigos, dos quais, após a leitura dos títulos e análise dos textos ficaram reduzidos a 8 .

A segunda busca foi realizada no site SCIELO que nos possibilitou encontrar 98 artigos, onde após a configuração dos filtros, critérios de inclusão e exclusão citados anteriormente e leitura dos textos, o número encontrado foi de 5 artigos adequados ao nosso tema, que resultou no final das 2 buscas, um total de 13 artigos. E para concluir, foi realizada uma busca na biblioteca virtual do Instituto Brasileiro de Medicina e Reabilitação (IBMR) e adicionado 1 livro. Totalizando 14 referências na construção desta pesquisa.

\section{Resultados e Discussão}

Para compor os resultados desta pesquisa foi elaborado uma síntese exposta no Quadro 1, com 3 tópicos: título, autor/ano de publicação e conclusão. Observa-se que todos artigos analisados datam dos últimos 5 anos 
Quadro 1: Apresentação da síntese dos artigos selecionados, Rio de Janeiro (RJ), Brasil, 2020.

\begin{tabular}{|c|c|c|c|}
\hline $\mathbf{N}^{\mathbf{o}}$ & Título do artigo & Autor/Ano & Conclusão \\
\hline 01 & $\begin{array}{lcr}\text { Cuidados } & \text { Paliativos } & \text { do } \\
\text { Enfermeiro } & \text { ao } & \text { Paciente } \\
\text { Oncológico. } & & \end{array}$ & $\begin{array}{l}\text { BRANDÃO, M. C. } \\
\text { P.; et al., } 2017\end{array}$ & $\begin{array}{l}\text { Os cuidados paliativos aplicados a pacientes oncológicos exigem } \\
\text { uma assistência sistematizada, tornando-se fundamental a } \\
\text { implementação da formação permanente aos enfermeiros. }\end{array}$ \\
\hline 02 & Cuidados paliativos & $\begin{array}{l}\text { GOMES, A. L. Z.; } \\
\text { OTHERO, M. } \\
2016\end{array}$ & $\begin{array}{l}\text { O cuidado paliativo tem conquistando espaço no Brasil, devido a } \\
\text { incidência de doenças crônicas degenerativas e do envelhecimento } \\
\text { da população. }\end{array}$ \\
\hline 03 & $\begin{array}{l}\text { O Envelhecimento Populacional } \\
\text { Brasileiro: } \begin{array}{l}\text { Desafios } \\
\text { consequências }\end{array} \text { sociais atuais } \mathrm{e} \\
\text { futuras }\end{array}$ & $\begin{array}{l}\text { MIRANDA, G. M. } \\
\text { D.; MENDES, A. C. } \\
\text { G.; SILVA, A. L. A., } \\
2016\end{array}$ & $\begin{array}{l}\text { Com o aumento do envelhecimento da população brasileira é } \\
\text { necessário reorganizar os níveis de cuidados especializados, } \\
\text { inovando para poder atender as novas demandas que estão por vir. }\end{array}$ \\
\hline 04 & $\begin{array}{l}\text { Assistência de Enfermagem e o } \\
\text { Enfoque da Segurança do } \\
\text { Paciente no Cenário Brasileiro }\end{array}$ & $\begin{array}{l}\text { SILVA, A. T.; et al, } \\
2016\end{array}$ & $\begin{array}{l}\text { O preparo dos profissionais na assistência de enfermagem é } \\
\text { fundamental para um atendimento seguro e de qualidade. }\end{array}$ \\
\hline 05 & $\begin{array}{l}\text { Percepções dos profissionais de } \\
\text { enfermagem acerca do uso da } \\
\text { informatização para segurança do } \\
\text { paciente }\end{array}$ & $\begin{array}{l}\text { FERREIRA, A.M.D.; } \\
\text { et al., } 2019\end{array}$ & $\begin{array}{l}\text { O uso da tecnologia de informação facilita a comunicação dos } \\
\text { profissionais de saúde auxilia e melhora o processo assistencial. }\end{array}$ \\
\hline 06 & $\begin{array}{l}\text { Cuidados paliativos, o cuidar de } \\
\text { uma forma humanizada }\end{array}$ & $\begin{array}{l}\text { COSTA, J. } \quad \text { B. } \quad \text { F.; } \\
\text { ASSUNÇÃO, T. } \\
\text { O.; SALLES, H. } \\
\text { A., } 2017\end{array}$ & $\begin{array}{l}\text { A comunicação entre profissionais, paciente e familiares deve ser } \\
\text { construída com base numa assistência humanizada por } \\
\text { profissionais especializados e preparados nas ações paliativas. }\end{array}$ \\
\hline 07 & $\begin{array}{l}\text { Teoria das Relações Interpessoais } \\
\text { de Peplau: Uma avaliação } \\
\text { baseada nos critérios Fawcett }\end{array}$ & $\begin{array}{l}\text { FRANZOI, M. A. H.; } \\
\text { et al., } 2016\end{array}$ & $\begin{array}{l}\text { A teoria de Peplau favorece a atuação do enfermeiro no } \\
\text { atendimento humanizado, utilizando a comunicação como base da } \\
\text { relação interpessoal entre as equipes, pacientes e familiares. }\end{array}$ \\
\hline 08 & $\begin{array}{l}\text { Comunicação Interpessoal com } \\
\text { Pacientes Oncológico em } \\
\text { Cuidados Paliativos }\end{array}$ & $\begin{array}{l}\text { GALVÃO, M. I. Z.; } \\
\text { BORGES, M. S.; } \\
\text { PINHO, D. L. M., } \\
2017\end{array}$ & $\begin{array}{l}\text { O foco principal da intervenção foi a correlação da teoria de Peplau } \\
\text { aos cuidados paliativos e a relevância da competência } \\
\text { comunicacional. }\end{array}$ \\
\hline 09 & $\begin{array}{l}\text { O Uso de Estratégias de } \\
\text { comunicação em Cuidados } \\
\text { Paliativos no Brasil }\end{array}$ & $\begin{array}{l}\text { ALMEIDA, K. L. S.; } \\
\text { GARCIA, D. M, } 2015\end{array}$ & $\begin{array}{l}\text { As estratégias de comunicação são métodos terapêuticos em razão } \\
\text { dos quais o profissional de saúde necessita ser capacitado nesta } \\
\text { temática. }\end{array}$ \\
\hline 10 & $\begin{array}{l}\text { Palliative Care In Brasil: Present } \\
\text { and Future }\end{array}$ & $\begin{array}{l}\text { SANTOS, C. E.; et } \\
\text { al., } 2019\end{array}$ & $\begin{array}{l}\text { Devido ao aumento de expectativa de vida e acessão das doenças } \\
\text { crônicas como o câncer no país, torna-se urgente uma estratégia } \\
\text { nacional em todos os níveis de atendimento e de serviços que } \\
\text { respondam a essa demanda. }\end{array}$ \\
\hline 11 & $\begin{array}{l}\text { Palliative Care and the } \\
\text { Importance of Communication } \\
\text { Between Nurse and Patient, } \\
\text { Family and Caregiver }\end{array}$ & $\begin{array}{l}\text { ANDRADE, G. B.; et } \\
\text { al., } 2019\end{array}$ & $\begin{array}{l}\text { A importância da comunicação nos cuidados paliativos e os } \\
\text { benefícios para a saúde física e psicológica do paciente. }\end{array}$ \\
\hline 12 & $\begin{array}{l}\text { Produção científica da } \\
\text { Enfermagem sobre promoção de } \\
\text { saúde, condição crônica e } \\
\text { envelhecimento }\end{array}$ & $\begin{array}{l}\text { VALCARENGH, R. } \\
\text { V.; et al, } 2015\end{array}$ & $\begin{array}{l}\text { Destaca a importância da família no cuidado com o idoso, e a } \\
\text { formação dos profissionais para promover o bem-estar do paciente } \\
\text { e seus familiares. }\end{array}$ \\
\hline 13 & $\begin{array}{l}\text { Cuidados Paliativos: Alternativa } \\
\text { para o Cuidado Essencial no Fim } \\
\text { da Vida. }\end{array}$ & $\begin{array}{l}\text { ALVES, R. S. F.; et } \\
\text { al., } 2019\end{array}$ & $\begin{array}{l}\text { Que as políticas públicas envolvendo os cuidados paliativos } \\
\text { necessitam ser trabalhadas assim como a preparação dos } \\
\text { profissionais que atuam nessa especialidade. }\end{array}$ \\
\hline
\end{tabular}

Fonte: Autores. 
$\mathrm{Na}$ apresentação dos resultados, segundo análise dos 13 artigos selecionados, verificou-se que eles apontam a comunicação como a base do atendimento da equipe de enfermagem nos cuidados paliativos (artigos $n^{\circ} 6,7,10,12$ ) e que existe um despreparo com o déficit de conhecimento das estratégias de comunicação e dos cuidados relacionados ao tema, devido uma falta de formação acadêmica e de capacitação dos profissionais (artigos nº 1,4,10,13-14).

Também podemos observar que o envelhecimento e as doenças crônicas como o câncer, são indicados no cuidado paliativo e a necessidade do envolvimento governamental na criação de políticas públicas e nos serviços especializados (artigos $\left.\mathrm{n}^{\circ} 1,2,3,11\right)$. A teoria da relação interpessoal de Peplau orienta a prática da assistência focando na comunicação, atendimento humanizado e no trabalho multidisciplinar para os cuidados paliativos ( $\operatorname{artigos} n^{\circ} 7$ e 9).

E para finalizar, o envolvimento da família no cuidado com idoso portador de câncer é essencial para o bem-estar do paciente e o sucesso do tratamento, sem esquecermos de que a família também necessita igualmente do cuidado e apoio dos profissionais da saúde (artigos n $\mathrm{n}^{\circ} 1,3,13$ ).

Em 1970, o Brasil era considerado um país jovem, mas com mudanças no perfil demográfico essa estatística mudou radicalmente. Essa mudança em razão do avanço da medicina e da redução das taxas de mortalidade e de natalidade, o país passou a ter mais idosos e menos jovens (Miranda, Mendes \& Silva, 2016).

Outrora, as doenças contagiosas eram motivo de um grande número de óbito, hoje, são as doenças crônicas não transmissíveis as principais causas de mortes (Almeida \& Garcia, 2015).

A Organização Mundial de Saúde (OMS) lista quatro tipos de doenças que mais matam anualmente e correspondem a $70 \%$ das mortes, no mundo. São elas: a diabetes; o câncer; as doenças respiratórias e circulatórias. As Doenças Crônicas Não Transmissíveis (DCNT), normalmente necessitam dos CP - por não terem cura - não busca adiar a morte, mas dar uma oportunidade do paciente viver melhor a vida (Santos et al, 2019).

A OMS, considera ainda, que todas essas doenças necessitam de uma estratégia continuada de política pública voltada para a saúde, no que diz respeito a prevenção e controle. Todos os esforços para desenvolver essas estratégias ainda não são eficazes, visto que o número de doentes portadores de DCNT teve um aumento significativo, nessa última década. No estudo Internacional da Observatory on End Of Live Care (IOELC), que teve início em 2003, na Universidade de Lancaster, mostra que 234 países, 15\% tinham uma política de qualidade voltada para os CP e 33\% não a adotavam. (10) Pesquisas apontam, que os países que inserem os $\mathrm{CP}$, na sua maioria, são voltados para os pacientes que tenham doenças neoplásicas crônicas. Contudo, em 2004 a OMS insere a necessidade do CP como parte da assistência aos pacientes com doenças crônicas e aos idosos (Gomes \& Othero, 2016).

As atividades relacionadas à prática dos $\mathrm{CP}$ ainda, passam por grandes dificuldades de aceitação, até mesmo preconceito, por ser uma área desconhecida por muitos. Há uma grande necessidade de implantação de modelos que gerem a padronização para um atendimento humanizado com eficiência e qualidade (Santos et al, 2019).

O estudo mostra que para efetivar a prática do cuidado, as estratégias de comunicação são indispensáveis para os profissionais da saúde, uma vez que se caracterizam pela habilidade em ajudar a pessoa a enfrentar situações adversas, lidar com o outro, diminuir a ansiedade, promover conforto e assim proporcionar uma assistência holística, de qualidade e humanizada (Andrade et al., 2019)

A comunicação possui dois eixos: a verbal e não verbal. A comunicação verbal se passa por meio da expressão falada e escrita, por meio dela o profissional pode se apropriar de várias estratégias para criar um relacionamento interpessoal com o paciente e seus familiares. Tendo como exemplo, estabelecer com o paciente e seus familiares metas e planos de ação, utilizar linguagem coloquial, com vocabulário simples, valorizar todas as informações dadas pelo paciente e pelo familiar, estimular a verbalização de medos, receios e angústias, oferecer tempo, verbalizar disposição para a atenção e cuidado, estabelecer opções e indicar diferentes pontos de vista (Almeida \& Garcia, 2015). 
Já na escrita, os registros de enfermagem e o sistema informatizado asseguram a comunicação entre profissionais de enfermagem e garantindo os bons resultados da assistência (Silva et al, 2016).

A comunicação não verbal é a que qualifica a expressão falada e está relacionada a todas as palavras não ditas ou expressas - pela fala. Ela é caracterizada por gestos, tom de voz, postura corporal, olhares, expressões, pelo toque e escuta ativa (Almeida \& Garcia, 2015).

No cuidado de idoso com câncer, a importância da atuação do profissional de enfermagem na ação educativa e na promoção do cuidado está ligada na relação interpessoal paciente/família. Diante disso, a família tem um papel fundamental no processo mental e psicológico do idoso, uma vez que assegura o enfrentamento da doença e suas adversidades, pelos os laços afetivos atenuando o sofrimento. O envelhecimento provoca alteração na capacidade funcional e cognitiva, exigindo do profissional, habilidades e conhecimentos para lidar com as pluralidades das situações que se apresentam. Assim, os programas de capacitação permanente para os profissionais tornam-se essenciais e exigem uma mudança no paradigma. Nas últimas décadas, as prioridades do Pacto em Defesa da Vida do Sistema Único de Saúde (SUS), estão incorporadas aos cuidados especializados e promoção de saúde para o envelhecimento, devido ao número crescente dessa população (Valcarengh et al, 2015).

O caminho de visibilidade que o cuidado paliativo encontrou, foi através das políticas públicas de humanização. O Ministério da Saúde (MS), buscando melhorar qualidade nas práticas do atendimento do SUS iniciou um programa de humanização de saúde através do programa nacional de humanização da atenção hospitalar (PNHAH). A iniciativa foi desencadeada a partir de um levantamento feito pelo MS que revelou a insatisfação dos usuários com os serviços de saúde. Constataram a falta de atenção e da incompreensão de alguns profissionais, as longas esperas por um atendimento, a falta de medicamento e insumos, confirmando uma relação desumana no que seria constitucional. A política nacional de humanização (PNH), existe para efetivar os princípios do SUS e se tornou a principal diretriz para as ações paliativas, atuando nas intervenções humanizadas, no cuidado do ambiente, na humanização da velhice e na comunicação, objetivando o respeito e oferecendo dignidade (Alves et al., 2019; Brasil, Ministério da Saúde, 2001).

É a partir do atendimento com o foco na humanização que surge a necessidade de reforçar as atitudes e os comportamentos por meio da comunicação. Ela vem ao encontro, por meio de estratégias de fortalecimento para a criação de uma maior interação e, até mesmo gerar vínculo na relação do cuidado, também no auxiliando o paciente no processo de enfrentar situações difíceis, afastando pensamentos e ideias negativas (Andrade et al., 2019; Silva et al, 2020).

$\mathrm{O}$ cuidado humanizado tem base na excelência e na empatia. As atividades do profissional de enfermagem vão além das técnicas da profissão e da administração de medicações. O enfermeiro(a) deve agregar às suas atividades: a psicologia, a linguagem (verbal ou não verbal), trabalhar o emocional e o espiritual de cada indivíduo de acordo com suas necessidades. Algumas estratégias são aplicadas para o apoio a família, como: oferecer total acesso a comunicação; incluí-los nas ações de cuidados; compreender as necessidades especiais de cada paciente e ter total controle dos sintomas gerados pela doença (Andrade et al., 2019).

Em um estudo realizado na Inglaterra por enfermeiras, foi constatado que a comunicação não era realizada de forma eficaz, trazendo angústia para os pacientes que não se sentiam seguros para falar de seus sentimentos. Não havendo uma comunicação eficiente entre todos os envolvidos, no processo dos CP, o tratamento e apoio prestado será ineficiente, devido a essa falta de interação (Almeida \& Garcia, 2015).

Profissionais da saúde relatam muitas dificuldades na construção da comunicação com pacientes, devido à falta de conhecimento aprofundado sobre a prática dos CP em virtude destes só se aplicarem aos pacientes que se encontrem em fase terminal. Em razão da falta do mesmo, muitos profissionais tendem a confundir as estratégias de comunicação com os sentimentos do doente. Quando a estratégia de comunicação é proposta de forma adequada, proporciona muitos benefícios ao 
tratamento. A música terapêutica é uma das estratégias, por exemplo, que pode ser usada com o intuito de acalmar a alma e remeter a boas lembranças, assim como aos bons sentimentos. Contudo, para isso é preciso aprimorar os conhecimentos através da educação continuada/aperfeiçoamento na profissão e sempre estar atento a tudo que envolve a área (Almeida \& Garcia, 2015; Prado \& Buiatti, 2016).

A teoria de Hildegard Peplau, sobre as relações interpessoais do enfermeiro (a) com pacientes, teve influência nas teorias da psicologia e psiquiatria surgindo assim a enfermagem psicodinâmica, que serviu de base para a elaboração dos conceitos, das fases e, até mesmo, o real papel do enfermeiro. Observe-se que ainda no atendimento da assistência de enfermagem, Peplau desenvolveu quatro fases que são: orientação, identificação, exploração e solução. Estas fases estão interligadas principalmente por estratégias de comunicação na relação interpessoal: a) Orientação - é o início da relação interpessoal. Ocorre quando o paciente busca a ajuda de um profissional. É a partir do primeiro contato que se determinará o grau de parceria entre eles - o que evidência a comunicação esclarecedora dos assuntos tratados. Esta fase é importantíssima para a melhor adesão ao tratamento. b) Identificação - é importante conhecer toda a equipe médica, ou seja, deve-se ter empatia e estabelecer vínculos de confiança. A comunicação interpessoal eficaz pode preencher as necessidades quando a afinidade é estabelecida pelo paciente. Deve-se oferecer espaço e voz ativa ao doente para a compreensão plena e integral com base no relacionamento sólido e de confiança. c) Exploração - após adquirir uma relação de confiança, o paciente utiliza estratégias de enfrentamento, que possam responder as suas inquietações e necessidades. Por meio de estratégias comportamentais e cognitivas, o indivíduo busca solucionar problemas ou equilibrar as emoções, controlar as ações perigosas, evitar os problemas e buscar apoio. As estratégias são elaboradas para que o paciente encontre forças para lutar contra a doença e as adversidades. d) Resolução - última fase do processo interpessoal, a fase da identificação e os problemas de dependência foram trabalhados para tornar o paciente mais forte e independente dos laços com o profissional. Os caminhos, desse binômio, devem se separar e a partir deste momento, novos objetivos devem ser traçados. Todos os profissionais envolvidos nos CP devem apoiar e contribuir para que o paciente potencialize seus pontos fortes e minimize suas debilidades com a autoconsciência e resoluções resilientes (Franzoi et al, 2016; Pinheiro et al, 2019).

Nas quatro fases de Hildegard Peplau, destacam-se diferentes papéis que são assumidos pelo enfermeiro(a). São eles: estranho, recurso, líder, professor e substituto. Desta maneira, no papel de estranho, enfermeiro e paciente são pessoas desconhecidas e por uma necessidade sentida do paciente, o enfermeiro o conduz a entender o que está acontecendo. Ambos trabalham juntos a fim de esclarecer e descobrir os problemas envolvidos na questão; como pessoa de recursos, o enfermeiro é o detentor das respostas para as possíveis dúvidas; no papel de líder, ele deve direcionar e dar seguimento as metas e ajudá-lo a alcançá-las; o de professor é a capacidade de informá-lo, educá-lo e motivá-lo para adquirir conhecimentos e produzir mudanças; e, por último, como conselheiro, ele deve escutar, responder as solicitações e opinar nas decisões do paciente (Franzoi et al, 2016; Galvão, Borges \& Pinho, 2017).

É importante assinalar que a comunicação eficaz dentro dos CP possibilita o levantamento das necessidades, desenvolve a empatia, a relação de ajuda e proporciona a capacitação voltada para o cuidado. Deve-se salientar também que as discussões em equipe são de grande valia para o desenvolvimento de ações práticas para o relacionamento interpessoal e aquisição das habilidades comunicacionais (Franzoi et al, 2016).

O resultado da análise demostra a necessidade de implantação de serviços que prestem assistência paliativa. Concernente a proposta para melhorar a assistência de enfermagem depreende-se que é a educação em serviço que deve ser um processo contínuo e permanente, em razão dos próprios enfermeiros reconhecerem o despreparo diante das exigências e a falta de conhecimento nos CP (Brandão et al, 2017). 


\section{Considerações Finais}

$\mathrm{O}$ resultado da pesquisa demonstra que o enfermeiro(a) tem um papel fundamental para pessoas diagnosticadas com câncer que se encontram em tratamento dos CP. Este por sua vez necessita ser promovido e instituído em todas as fases das doenças incuráveis e não somente na fase terminal do paciente.

Esta abordagem permite ao enfermeiro(a) assistir tanto ao paciente quanto sua família, a enfrentarem todos os obstáculos pelos quais o doente passará até o fim da vida e, principalmente, como ele pode lidar de forma adequada nas etapas do tratamento.

Foi possível mostrar que através da comunicação, que os vínculos afetivos são desenvolvidos e que as estratégias de comunicação devem ser adquiridas e desenvolvidas baseadas no cuidado humanizado e que uma educação continuada, é um fator imprescindível para que o resultado da assistência de enfermagem seja integral e bem-sucedida. Podemos também observar que no cuidado paliativo, a teoria interpessoal de Peplau contribui para atuação do enfermeiro visto que, se enquadra ao cuidado, devido sua base fundamentada na enfermagem psicodinâmica.

Os estudos analisados mostraram ainda, que o processo de comunicação entre os membros da equipe de enfermagem é desenvolvido em função do paciente e seus familiares e que os pacientes em cuidados paliativos, tratados por profissionais qualificados podem obter uma assistência desenvolvida em toda a sua plenitude, a partir dos benefícios gerados pelas estratégias de comunicação.

Como recomendação para trabalhos futuros, cita-se: A realização da mesma temática, porém com metodologia de pesquisa de campo, diretamente com os usuários, podendo ser feitas variações, com os profissionais da saúde, com os pacientes e/ou com a família dos pacientes.

\section{Referências}

Almeida, K. L. S. \& Garcia, D. M. (2015). O Uso de Estratégias de comunicação em Cuidados Paliativos no Brasil: Revisão Integrativa. Revista Cogitare Enfermagem, 20(4).

Alves, R. S. F., et al. (2019). Cuidados Paliativos: Alternativa para o Cuidado Essencial no fim da vida. Psicologia: Ciência e Profissão, 39.

Andrade, G. B., et al. (2019). Palliative Care and the Importance of Communication Between Nurse and Patient, Family and Caregiver. Revista de Pesquisa Cuidado é Fundamental Online, 11(3)

Brasil. Ministério da Saúde. (2001). Secretaria de Assistência à Saúde. Programa Nacional de Humanização da Assistência Hospitalar, Brasília - DF, 60p, 2001 .

Brandão, M. C. P., et al. (2017). Cuidados Paliativos do Enfermeiro ao Paciente Oncológico. Revista Brasileira de Saúde Funcional, Cachoeira-BA, 1(2).

Costa, J. B. F., Assunção, T. A. O. \& Salles, H. S. A. (2017). Cuidados paliativos, o cuidar de uma forma humanizada. Enfermagem Brasil, 16(1).

Ferreira, A. M. D., et al. (2019). Percepções dos profissionais de enfermagem acerca do uso da informatização para segurança do paciente. Revista Gaúcha de Enfermagem, 40 .

Franzoi, M. A. H., et al. (2016). Teoria das Relações Interpessoais de Peplau: Uma avaliação baseada nos critérios Fawcett. Revista de Enfermagem UFPE On Line, Recife, 10(Supl. 4): 3653-61.

Galvão, M. I. Z., Borges, M. S. \& Pinho, D. L. M. (2017). Comunicação Interpessoal com Pacientes Oncológico em Cuidados Paliativos. Revista Baiana Enfermagem, 31(3)

Gomes, A. L. Z. \& Othero, M. B. (2016). Cuidados paliativos. Estudos Avançados, 30(88): 155-166.

Karine, M. R. (2018). Princípios dos Cuidados Paliativos. Sahah Educação S.A, 21-24.

Ludke, M. \& Andre, M. E. D. A. (2013). Pesquisas em educação: uma abordagem qualitativa. E.P.U.

Merino, M. F. G. L., et al. (2018). Nursing theories in professional training and practice: perception of postgraduate nursing students. Revista da Rede de Enfermagem do Nordeste, 19.

Miranda, G. M. D., Mendes, A. C. G. \& Silva, A. L. A. (2016). Population aging in Brazil: current and future social challenges and consequences. Revista Brasileira de Geriatria e Gerontologia, 19(3): 507-519. 
Research, Society and Development, v. 10, n. 11, e333101119499, 2021 (CC BY 4.0) | ISSN 2525-3409 | DOI: http://dx.doi.org/10.33448/rsd-v10i11.19499

Pinheiro, C. W., et al. (2019). Teoria das Relações Interpessoais: Reflexões acerca da função terapêutica do Enfermeiro em Saúde Mental. Enfermagem em foco, 10(3):64-69.

Prado, C. G., Buiatti, V. P. (2016). Psicologia na Educação. Monografia - Curso de Licenciatura em Matemática. Universidade Federal de Uberlândia, Uberlândia - MG, 2016, 142p.

Santos, C. E., et al. (2019). Palliative Care In Brasil: Present and Future. Revista da Associação Médica Brasileira, 65(6).

Silva, A. T., et al. (2016). Assistência de Enfermagem e o enfoque da Segurança do Paciente no Cenário Brasileiro, Saúde em Debate, 40(111).

Silva, M. E., et al. (2020). Suicide Prevention Actions in Brazil. International Journal of Development Research, 10(10): 41034-41037.

Siqueira, P. L. F. (2020). Systematization of assistance, theories and nursing process - a literature review. Research, Society and Development, 9(10).

Valcarengh, R. V., et al. (2015). Produção científica da Enfermagem sobre promoção de saúde, condição crônica e envelhecimento. Revista Brasileira de Enfermagem, 68(4) 